\title{
Korpuslinguistik und Übersetzung von \\ Konnektoren. Zur Bedeutung von repräsentativen \\ Datensammlungen und korpusbasierten \\ sprachwissenschaftlichen Analysen \\ für die Übersetzungspraxis
}

Corpus Linguistics and the Translation of Connectives. On the Importance of Representative Data Collections and Corpus-based Linguistic Analyses for Translation in Practice

Iva Zündorf

\begin{abstract}
Numerous fields of linguistic research are now inconceivable without data analyzes in corpora. This also means that the creation of corpora is not meant to be an end in itself, but that it primarily has a serving function. The paper aims to discuss the potential of text-based corpus linguistic analyzes by means of authentic examples of argumentative connectives as well as to show the connection with translatological questions.
\end{abstract}

\section{Keywords}

corpus linguistics; translation; text configurations; connectives 


\section{Korpuslinguistik und Übersetzungswissenschaft}

Wie bereits der Untertitel andeutet, zielt der Artikel auf die Relevanz und Effizienz von korpuslinguistischen Methoden und Instrumenten bei der Lösung von translatologischen Fragen. Korpora im heutigen Sinne werden als strukturierte Sammlungen von Texten verstanden, die ohne Frage eine repräsentative Datengrundlage für unterschiedliche Arten linguistischer Untersuchungen darstellen. Dies ist aus heutiger Sicht aber nicht die einzige Systemfunktionalität dieser umfangreichen Datenquellen; aufgrund der Tatsache, dass die technischen Möglichkeiten der Datenverarbeitung immer effizienter und vor allem der Grundlagenforschung immer zugänglicher werden, stellen Korpora heutzutage generell eine natürliche Basis für die Entwicklung weiterer relevanter Instrumente dar, die für die Sprachwissenschaft sowie für die angewandten sprachwissenschaftlichen Disziplinen von Nutzen sind. Diese Entwicklung ermöglicht uns Fragen zu stellen, die wir mit traditionellen Datenquellen nicht so leicht hätten beantworten können, mit solchen Instrumenten, z. B. korpusbasierten Datenbanken, können wir die Optik unserer sprachgebrauchsorientierten Fragestellungen auch völlig anders fokussieren ${ }^{1}$.

Aus Erfahrung wissen wir, dass die allgemeine Einstellung zum Gebrauch von korpuslinguistischen Mitteln, vor allem in der Praxis, immer noch durch eine gewisse Zurückhaltung geprägt ist. Der Grund dafür kann natürlich an mehreren Faktoren liegen: Meistens ist es die Größe der Datenmengen, die Schwierigkeiten bereitet, eine große Rolle spielen auch mangelnde praktische Kompetenzen, die die Korpusrecherchen aber brauchen, damit man mit der richtigen Suchanfrage, mit entsprechenden Algorithmen und schließlich auch mit der entsprechenden Dateninterpretation zu einer guten Lösung kommen kann.

Korpora und korpusbasierte Instrumente stellen eine effiziente Quelle für Aufgaben dar, die Sprache im Gebrauch untersuchen; sie nehmen aber auch die verschiedenen Funktionen unter die Lupe, in denen Sprache in Erscheinung tritt. (Vgl. Perkuhn/ Keibel/Kupiec 2012: 14f.) Sowohl für die Übersetzungspraxis als auch für die Übersetzungswissenschaft sind beide Punkte wichtig, bei Entscheidungsprozessen über die Adäquatheit translatologischer Referenzinstrumente müssen sie im gleichen Maße berücksichtigt werden.

Bleiben wir jedoch bei unserer Grundfrage: In welchem Bezug stehen nun die sprachwissenschaftlichen Forschungsaufgaben und die translatologisch relevanten Fragestellungen?

Die Grenze zwischen kontrastiven und übersetzungsausgerichteten Analysen verläuft fließend. Zur Feststellung der anderssprachigen Äquivalenz, sowohl der grammatischen als auch der lexikalischen, ist es immer notwendig, das jeweilige Sprachelement im Kontext zu betrachten. Denn, wie Wolfgang Teubert formuliert, [...] „dass die Bedeutung der Wörter Textbelegen zu entnehmen ist, war seit Adelung und Campe das Grundprinzip

1 Hierzu Kratochvílová-Zündorf 2010: 171 
der klassischen Lexikographie, das von der Korpuslinguistik übernommen wird. “ (Teubert 1999: 302).

Es ist natürlich nicht möglich, an dieser Stelle die ganze Typologie von Korpora detailliert zu diskutieren, es ist auch nicht nötig. Für die weiteren Ausführungen werden insbesondere die folgenden Korpora in Betracht gezogen:

1. Die monolingualen Referenzkorpora als Instrumente, die in Größenordnung einige Milliarden Wörter umfassen. Hierzu gehören:

a) Die IDS-Korpora ${ }^{2}$, ein bedeutendes Beispiel dafür, wie man mit modernen technischen Mitteln Sprache für die Forschung und für weitere Instrumente aufbereiten kann. Trotz der monolingualen Ausrichtung ist das DeReKo, mit aktuell über 42 Milliarden Wörtern ${ }^{3}$, sowohl für die Sprachwissenschaft als auch für die Übersetzungswissenschaft eine bedeutende Datenquelle, wobei für die zweite auch indirekt, vor allem durch die auf ein Referenzkorpus basierten Datenbanken und Portale, die zur Lösung von übersetzungsrelevanten Fragen beitragen können. Darauf komme ich später noch mit konkreten Beispielen zurück.

b) Das tschechische Referenzkorpus bzw. das Tschechische Nationalkorpus ČNK, wurde am Institut für das Tschechische Nationalkorpus (ÚČNK Praha) in den 90er Jahren erstellt und wird weiter aufgebaut, die aktuellen Datenangaben belaufen sich auf 4 Milliarden ${ }^{4}$. In Studien zum deutsch-tschechischen Sprachvergleich wird am häufigsten auf das mehrsprachige Parallelkorpus Intercorp zurückgegriffen, das in der neuesten Version Intercorp9 mittlerweile eine beachtliche Menge von Paralleltexten in 39 Sprachen zur Verfügung stellt, davon 23 getaggte Sprachen und 20 lemmatisierte Sprachenpaare $^{5}$. Jeder Text ist im Korpus mit der entsprechenden tschechischen Version aligniert (als Original oder Übersetzung). Als Option kann man die Namen der Autoren sowie der Übersetzer abrufen, in der direkten Recherche werden sie nicht angezeigt.

2. Parallelkorpora generell sind translatologisch relevant. Nicht etwa als Zettelkasten oder Wörterbuch, dafür sind, wie auch z. B. bei Intercorp, in einigen Texten die Spuren der Übersetzung sichtbar und die Visualisierung des Originals erfolgt leider nicht automatisch. Für translatologisch relevante Fragen aber, die sich grundsätzlich am WIE orientieren, d.h. mit welchen übersetzerischen Strategien der Text in die Zielsprache übertragen worden ist, muss das nicht unbedingt von Nachteil sein. Im Gegenteil, sprachenpaarspezifische Übersetzungsprobleme können mit diesem Instrument sehr gut erfasst und untersucht werden. (Vgl. Kratochvílová 2013: 16f.).

2 Das Institut für deutsche Sprache in Mannheim mit seiner Arbeitsstelle für linguistische Datenverarbeitung stellt die wichtigste zentrale Institution im deutschsprachigen Raum dar, die über Korpus-Ressourcen verfügt. (Vgl. Teubert 1998: 147).

3 http://www1.ids-mannheim.de/kl/projekte/korpora.html (28. 6. 2018)

4 https://www.korpus.cz/ (28. 6. 2018)

$5 \quad$ Nach wiki.korpus.cz (18. 3. 2017) 
Den Translatologen, und vor allem den Translatologie-Studierenden, steht somit wertvolles Material zur Verfügung, an dem sie praktische Probleme der Übersetzung identifizieren und analysieren können.

Bei sprachwissenschaftlichen Fragestellungen, d.h. bei Fragen danach WAS einer ausgangssprachlichen Struktur entspricht, müssen wir bei Parallelkorpora die Übersetzungsrichtung auf jeden Fall berücksichtigen. Eigene Erfahrungen mit linguistisch- bzw. textorientierten Korpusrecherchen bringen mich zur Erkenntnis, das ausgewogene bi-direktionale Korpora, in denen ganze Texte, nicht nur Textabschnitte, exhaustiv recherchiert werden können, in der Phase der Hypothesenbildung sehr gut funktionieren ${ }^{6}$.

Für die frequenzorientierten Nachuntersuchungen, falls sie für die Forschungsaufgabe nötig sind, muss man dann entsprechende Teile der Referenzkorpora - als Subkorpora - heranziehen. Der methodische Vorgang wurde bereits beschrieben, insbesondere in Arbeiten der Projektgruppe Korpuslinguistik Deutsch-Tschechisch kontrastiv, die sich intensiv mit der Problematik der sog. kleinen Korpora beschäftigt hat und auch zu plausiblen Ergebnissen gekommen ist. ${ }^{7}$

3. Übersetzungsrelevant sind in gleicher Weise die Vergleichskorpora oder Kontrastkorpora. Diese bestehen aus Texten in zwei oder mehreren Sprachen, synoptisiert werden sie jedoch aufgrund ihrer Vergleichbarkeit nach dem festgesetzten ,tertium comparationis - so etwa weil sie derselben Textsorte angehören. Es handelt sich jeweils um Originaltexte, die z. B. für die terminologische Extraktion oder für Fragen der Diskursforschung geeignet sind.

\section{2. Ëinzelsprachliche Konfigurationen, Sprachgebrauchsmuster und Übersetzung}

Die Praxis hat gezeigt, dass es vor allem einzelsprachlich spezifisch ausgeprägte Sprachgebrauchsmuster $^{8}$ sind, die bei der Übersetzung Schwierigkeiten bereiten, seien es systemische Mehrwortverbindungen, Kollokationen oder spezielle Konfigurationen oder sogar sprachenspezifische Konfigurationen auf der Textebene. Beide haben eins gemeinsam - die einzelsprachliche Typik, die durch das fremdsprachliche Register häufig nicht adäquat reflektiert wird.

Zur sprachlichen Typik gehören in erster Linie die Kollokationsstrukturen. Sie fungieren als eigenständige „lexikalisch-syntaktische Standards, die als rekurrente Kombinationen von sprachlichen Strukturmustern und lexikalischer Besetzung in spezifischen Kommunikationssituationen zur Vermittlung bestimmter sprachlicher Handlungen eingesetzt werden“" (Rothkegel 1994: 516).

6 Mehr dazu in Kratochvílová-Zündorf (2013: 17f.)

7 Siehe Kratochvílová-Zündorf, I./N.R. Wolf (Hg.) Kompendium Korpuslinguistik (2010)

8 Im Sinne von Bubenhofer (2009) 
Translatologisch gesehen, verstehen wir die Kollokationsstrukturen als selbständige Übersetzungseinheiten, da sie als mehr oder weniger feste oder konfigurierte Sprachgebrauchsmuster in Texten eingesetzt werden. Sie sind vor allem bei solchen Übersetzungen wichtig, in denen ein pragmatisch stabilisiertes Wissen vorausgesetzt wird $^{9}$, nicht nur im Bereich der Terminologie, sondern generell ein stabilisiertes Wissen über den z. B. fachbezogenen Sprachgebrauch.

Eine intuitive Entscheidung über die Adäquatheit der jeweiligen Konfiguration ist häufig nicht ausreichend, die zweisprachigen Lexika bieten bei translatologischen Entscheidungsprozessen über usuelle oder fachspezifische Mehrwortverbindungen keine zuverlässige Stütze. Dies betrifft die deutsch-tschechische Übersetzungspraxis in erheblichem Maße, da die zugänglichen tschechisch-deutschen Wörterbücher bei Fragen der Kollokationen immer noch über wesentliche Mängel verfügen.

In diesem Bereich sehen wir in den letzten Jahren doch eine positive Entwicklung, die von Seiten der Korpuslinguistik gekommen ist. Die Kollokationsforschung, sowohl korpusgeleitet als auch korpusbasiert, ist seit einigen Jahren zu einer selbständigen Disziplin geworden. Die Konsequenz ist, dass fast jede sprachwissenschaftlich orientierte Korpusrecherche es in den Referenzkorpora ermöglicht, die signifikanten Wortverbindungen im Kontext zu erfassen und weiter korpuslinguistisch zu untersuchen.

Die usuellen Mehrwortverbindungen und Kollokationsstrukturen sind aber nicht das einzige Problem, mit dem die Übersetzungspraxis konfrontiert wird. Die einzelsprachlich spezifischen Sprachgebrauchsmuster gehen über die Wortgrenze hinaus bis auf die Textebene. Das textlinguistische Wissen, d.h. das Wissen über die jeweilige Textsorte und ihre Textaufbauprinzipien, Konventionen in der Gedankenführung (vgl. Blumenthal 1997:113) sollen daher zu den wichtigen Grundlagen des translatologischen Wissens gehören.

\subsection{Spezialfall: Transphrastische Konfigurationen und Sprachgebrauchsmuster}

Eine wichtige Rolle für den Textaufbau über die Satzgrenze hinaus spielen die sprachlichen Elemente, die Konnektoren, die für die Sprachwissenschaft, und für die Übersetzungswissenschaft vielleicht noch mehr, von Interesse sind und eine große Herausforderung darstellen.

Die Konnektoren verstehen wir als Funktionszeichen, die im Text „die Art der semantischen Relation signalisieren, welche zwischen zwei aufeinanderfolgenden Äußerungen hergestellt werden soll.“ (Breindl 2011: 31). Es handelt sich um „Konjunktionen und Konjunktionaladverbien, bisweilen auch feste Zeichenfolgen (etwa kurz und gut), die die semantische Relation zwischen zwei Sätzen signalisieren." (Wolf 2008:63f.) Wir bleiben

9 Vgl. Kratochvílová (Zündorf) 2015: 75f. 
in den weiteren Ausführungen bei dieser semantisch-funktionalen Auffassung von Konnektoren ${ }^{10}$ und unterscheiden demnach folgende Gruppen:

a) Situative Relation: dann, da, danach, so, nun, unterdessen (Zeit, Situation)

b) Anreihende/erweiternde Funktion: und, auch, ferner, übrigens, überdies

c) Spezifizierende und hervorhebende Funktion: und zwar, außerdem, vor allem

d) Zusammenfassende Funktion: kurzum, jedenfalls

e) Argumentative Relationen: KAUSAL, ADVERSATIV

Widmen wir uns nun den beiden Untergruppen der argumentativen Konnektoren etwas ausführlicher:

I. KAUSALE Relationen werden bei Begründungen oder Folgerungen mit Hilfe von Konnektoren versprachlicht. Hierzu gehören beispielsweise:

a) Begründung: denn, nämlich, deshalb

b) Folgerung: so, also, daher, darum, demnach, demzufolge, deshalb, deswegen, folglich, infolgedessen, mithin, somit

II. ADVERSATIVE Relationen drücken, wie aus den Beispielen ersichtlich, Alternative, Gegensatz, Einschränkung oder Einräumung aus:

a) Alternative: oder, sonst

b) Gegensatz: aber, dennoch, dagegen, indes, indessen

c) Einschränkung - Widerspruch: jedoch, allerdings, wohl

d) Einräumung (konzessiv): dennoch, des(sen) ungeachtet, gleichwohl, immerhin, trotzdem, nichtsdestoweniger, nichtsdestotrotz, zwar...aber u.a.

Wie aus dieser Übersicht ersichtlich, drückt jeder Konnektor eine bestimmte logische Relation aus, gleichzeitig erfüllen diese Funktionszeichen im Text auch eine „allgemeine Steuerungs- und Orientierungsfunktion“ (Schanen 2001: 12). Der adäquate Gebrauch von Konnektoren in entsprechenden Kontexten reflektiert die Merkmale der einzelsprachlichen Typik, einige mehr oder weniger feste Verbindungen mit Konnektoren manifestieren die typische Gedankenverkettung und Argumentation der Textproduzenten einer Sprache.

Die Übersetzer stoßen in beiden Übersetzungsrichtungen (bei der Übersetzungsrichtung Tschechisch-Deutsch doch vielleicht noch mehr) auf das Problem der Nivellierung von diesen wichtigen Funktionszeichen (in der Übersicht mit Unterstreichungen markiert). Der Grund ist die offenbare pragmatische Unsicherheit über die textuelle Funktion dieser Zeichen. Übersetzungsstrategisch werden sie nun häufig durch Simplifikationen oder auch auf Grund von Levelling durch allgemein bekannte Konnektoren ersetzt - wie z. B. im Falle der adversativen Relationen bei indessen bzw. indes oder bei konzessiven gleichwohl, nichtsdestoweniger, nichtsdestotrotz.

10 Siehe Wolf 1981: 209f. 


\section{Das Potential der korpuslinguistischen Instrumente bei translatologischen Fragen}

Aus translatologischer Sicht stellen sich hier außer Fragen der erwartbaren Äquivalenz noch weitere Problemstellungen:

a) Mit welchen Instrumenten können wir die funktionale Distribution von möglichen Äquivalenten im Text feststellen?

b) Kann das gewählte Instrument helfen, die Frage nach dem WARUM funktional zu beantworten?

Als Instrument zur Lösung dieser Fragen wurde generell das ČNK und sein Parallelkorpus Intercorp herangezogen bzw. die korpusbasierte Datenbank Treq. Treq ist eine relativ neue Applikation des ČNK, sie steht seit 2014 zur Verfügung und wird auch weiterhin entwickelt und erweitert. Die neueste Version Treq 2.0. ermöglicht schon die Suche nach Mehrwortverbindungen. Die folgende Übersicht zeigt Instrumente des ČNK. Relevant für die Fragestellung dieser Studie sind insbesondere die Module $S y D$ und Treq.

\begin{tabular}{|ll}
\hline Kontext & $\begin{array}{l}\text { Frequenz, Konkordanzen, Assoziationsmaße, } \\
\text { Kollokationen }\end{array}$ \\
morfio & $\begin{array}{l}\text { Textinterpertation - Schlüsselwörter im } \\
\text { konkreten Text (Vergleich mit Korpusdaten) }\end{array}$ \\
SyDWOrdS & $\begin{array}{l}\text { Instrument für den synchronen sowie } \\
\text { diachronen Vergleich von Varianten } \\
\text { (Synonymie) }\end{array}$ \\
Treq & korpusbasierte Übersetzungsdatenbank \\
\hline
\end{tabular}

Abb.1 Übersetzungsrelevante Instrumente des ČNK

Die quantitative Analyse in Treq hat gezeigt, dass für indessen zwei differierende Lesarten funktional sind; die temporale Lesart als Ausdruck der Gleichzeitigkeit mit dem tschechischen Äquivalent zatím, mezitím und die adversative Lesart, übersetzt als však, ovšem. Durch die qualitative Interpretation der Kontextbelege kann der Übersetzer entscheiden, wie die Bedeutungen funktional distribuiert werden - dieser Schritt hilft bei der Entscheidung, dass es sich im Falle von indessen überwiegend um bildungssprachliche Kontexte handelt. Die Äquivalenzanalyse mit Hilfe dieses korpusbasierten Instruments brachte auch weitere gewichtige Anregungen. 
Die Äquivalenzreihe zum tschechischen však, wofür das deutsche Segment als erste Entsprechung jedoch vorschlägt, brachte eine interessante Reihe, die unsere Beispielsammlung von übersetzerischen Zweifelsfällen erweitert, mit nichtsdestoweniger, nichtsdestotrotz. Differenzierende Entsprechungen für nichtsdestoweniger, nichtsdestotrotz, sind in der Übersetzungsrichtung Tschechisch-Deutsch nicht belegt. Die pauschale Übersetzung mit presto oder nicméně soll nun mit weiteren korpusbasierten Instrumenten überprüft werden.

Wechseln wir deshalb die Übersetzungsrichtung und versuchen, mit systemgrammatischen Mitteln über das tschechische Funktionswort nicméně etwas mehr zu erfahren: Nach neueren tschechischen Grammatiken ist nicméně eine adversative Konjunktion, die Propositionen A und B verbindet, beide sind wahr, B ist jedoch im Widerspruch mit der Erwartung, die man mit A verbindet - B korrigiert dann diese Erwartung. Die Lesart kann jedoch auch als konzessiv betrachtet werden, nicméně verglichen mit přesto oder ale wirkt in der Interaktion sanfter. Dem tschechischen nicméně wird in Texten auch eine besondere kohärenzstiftende Funktion zugeschrieben, durch die die stilistische Markierung ,gehoben' neutralisiert wird. Als adverbiale kommentierende Partikel (vgl. Slezáková 2002: 199 und Štícha 2003: 132) ${ }^{11}$ fungiert nicméně insbesondere in der Initialposition als Mittel der Anknüpfung.

Für die weitere Suche im ČNK wurde das Variantenmodul SyD gewählt (siehe Abb.1), das den Vergleich von nicméně und přesto belegen sollte. Die SyD-Recherche ${ }^{12}$ zeigte die Zunahme von nicméně im gesprochensprachlichen Usus, kennzeichnend ist auch die Präsenz der jüngeren Generation sowie der höhere Bildungsgrad. Auch wenn wir annehmen, dass nichtsdestoweniger oder nichtsdestotrotz dem tschechischen nicméně im Kriterium 'häufiger Einsatz in der Interaktion' ebenfalls funktional entsprechen können, müssen wir weitere korpusbasierte Instrumente heranziehen, um die beiden sprachlichen Zeichen differenzieren zu können. Als Prämisse nehmen wir die folgenden korpuslinguistisch und lexikographisch relevanten Argumente:

a) Vorkommen - nach neueren Korpusuntersuchungen ist die Frequenz von nichtsdestotrotz verglichen mit nichtsdestoweniger hoch, im Verhältnis von etwa $4: 1^{13}$

b) Konventionalität - Duden bezeichnet nichtsdestotrotz als umgangssprachlich ${ }^{14}$, wohl aufgrund einer ursprünglich scherzhaften Mischbildung von nichtsdestoweniger und trotzdem.

Mehr Informationen zu diesem Vergleich gewinnen wir mit weiteren korpusbasierten Instrumenten aus der Projektreihe des IDS:

11 Hierzu auch Macháčková, Eva: Renesance výrazu nicméně, Ň̌ 73, 1990, s. 219-220.

12 Vergleich nicméně und přesto im Variantenmodul SyD abrufbar unter https://syd.korpus.cz/ (20. 3. 2018)

13 Vgl. Wassner 2011: 69

14 Vgl. https://www.duden.de/suchen/dudenonline/nichtsdestotrotz (20. 3. 2018) 
1. Eine Möglichkeit bietet die Analyse von semantisch nahen Kookkurrenzprofilen in der Kookkurrenzdatenbank CCDB ${ }^{15}$. Dieses Instrument zeigt, wie die Bedeutungen distribuiert sind, welche Wörter in der jeweiligen Umgebung der untersuchten Ausdrücke semantisch vergleichbar sind und welche dagegen nicht. Die visuelle Ausgabe der Kookkurrenzen, farblich (rot und gelb) markiert, ermöglicht eine schnelle Orientierung. Bei nichtsdestotrotz verglichen mit nichtsdestoweniger finden wir zahlreiche Treffer, die auf Gesprochensprachlichkeit hinweisen, dagegen aber auch Ausdrücke, die dem schriftlichen Ausdruck entsprechen ${ }^{16}$.

Als Instrument der ersten Wahl für translatologisch relevante Fragen ist vor allem „ein korpusbasiertes Online Informationssystem zur deutschen Gegenwartssprache“ elexiko zu empfehlen, „das den Wortschatz der deutschen Sprache anhand von aktuellen Sprachdaten [...], erklärt und wissenschaftlich kommentiert [... “"17

Das Online Instrument e-lexiko verweist zwar auch auf grammatische Angaben, bei der Suche nach grammatischer Beschreibung von Konnektoren zeigte sich jedoch das grammatische Portal Grammis als besser geeignet. Hier finden wir eine Übersicht der Positionsmodalitäten. Wie die Beispiele zu nichtsdestotrotz ${ }^{18}$ veranschaulichen, fungiert dieses Funktionswort als ein nichtpositionsbeschränkter Adverbkonnektor mit konzessiver Bedeutung:

Vorfeld: Das klingt bis hierhin ziemlich albern, nichtsdestotrotz hätte aus dem Film ein echter Tränenräuber werden können. (Berliner Zeitung, 25. 11. 2000, S. 20)

Mittelfeld: Alles, was aus den eigentlich zu versteuernden Gewinnen in diese Rückstellungstöpfe weggedrückt wird, braucht nicht versteuert, kann aber nichtsdestotrotz zinsbringend angelegt werden. (die Tageszeitung, 27. 2. 1987, S. 8)

Nullposition: Die jungen Zuschauer ab vier Jahren beobachteten zwar verblüfft, wie diese Menschen auf der Bühne Nachspringen spielten. [...] Nichtsdestotrotz, zum Schluss wurde eifrig geklatscht. (Mannheimer Morgen, 8. 11. 1989, o. S.)

Die grammatischen Angaben sollten bei translatologischen Entscheidungsprozessen durch qualitative Interpretationen authentischer Textvergleiche ergänzt werden. Dies ist bei diesem Instrument gut möglich, da elexiko bzw. das OWID-Portal auch eine vereinfachte Korpussuche ermöglicht:

15 Die Kookkurrenzdatenbank CCDB wurde „im Programmbereich Korpuslinguistik des Instituts für Deutsche Sprache auf der Grundlage eines Korpus geschriebener Gegenwartssprache von ca. 2,2 Milliarden laufenden Textwörtern als eine große Sammlung von Kookkurrenzprofilen zu ca. 220.000 verschiedenen Lemmata aufgebaut. Die Sammlung enthält zu jedem Lemma die Ergebnisse von bis zu fünf verschiedenen Kookkurrenzanalysen in Form von Hierarchien von ähnlichen Verwendungen, mit bis zu 100.000 Verwendungsbeispielen pro Lemma und Analyse." (Belica 2011 ff. http://corpora.ids-mannheim.de/ccdb/) (15. 3. 2018)

16 Vgl. Kookkurrenzprofile in 'contrast near synonyms' von nichtsdestoweniger und nichtsdestotrotz, http:// corpora.ids-mannheim.de/ccdb/

17 https://www.owid.de/wb/elexiko/start.html (20. 8. 2017)

18 https://grammis.ids-mannheim.de/konnektoren/407123 (20. 8. 2017) 
Interessant ist, dass die Zufallsauswahl der Korpussuche im elexiko häufige Fälle der Vorfeldposition oder der Nullposition visualisiert, wie die folgenden Beispiele auch zeigen:

Drittens ist zwar eigentlich eine Selbstverständlichkeit. Aber wenn ich den Minister jetzt höre: „Meiner Meinung nach ist das schon notwendig „, glaube ich, dass es schon sinnvoll wäre, das durch den Landtagsbeschluss noch zu erhärten. Es wäre sinnvoll, noch einmal festzustellen, dass, wenn Anteile des Landes der Bank zum Verkauf kommen, hier eine Entscheidung gefällt werden muss und dass wir erwarten, dass diese Entscheidung dann auch hier fällt.

Nichtsdestotrotz ist klar, wie ich vorhin schon ausgeführt habe: Sollten unsere sächsischen Sparkassen ihre Anteile verkaufen wollen, sind wir eh wieder aus dem Geschäft -um das noch einmal deutlich gesagt zu haben.

(Protokoll der Sitzung des Parlaments Sächsischer Landtag am 4. 7. 2007.83.

Sitzung der 4. Wahlperiode 2004-2009. Plenarprotokoll, Dresden, 2007 [S. 6904])

Dabei war dieses Recht erst im Februar eingeführt worden. Dieses Verfahren der Gesetzgebung sei durchaus ungewöhnlich, hieß es in Regierungskreisen. Auch manche Rechtsfrage zu den Neuregelungen blieb offen. Nichtsdestotrotz: Die Koalition wollte die Reform der Reform schnell verabschieden, weil die Regierung damit noch dieses Jahr 500 Millionen Euro und von 2007 an bis zu 1,5 Milliarden Euro jährlich sparen will. Das so genannte Fortentwicklungsgesetz zu Hartz IV, das am 1. August in Kraft treten soll, bringt gut 50 Änderungen - wie viele genau es sind, überblickt kaum mehr jemand. Die meisten Korrekturen bringen mehr Kontrollen und Strafmöglichkeiten.

(Süddeutsche Zeitung, 2. 6. 2006, S. 5; Größte Korrektur der Arbeitsmarktreform)

Eine noch deutlichere Angelegenheit wurde die Viertelfinalbegegnung zwischen den Teams Nordrhein-Westfalen 2 und Baden-Württemberg 29. Mahmoud Tabrizi (ZBB Weinheim) und Cedric Schubert (TSG Lützelsachsen) hatten hier nicht den Hauch einer Chance. Die beiden Bergsträßer verloren mit 0:13 und kassierten somit die „Höchststrafe“ eines jeden Petanque-Spielers.

Nichtsdestotrotz, das Fazit der Veranstaltung fiel fast einhellig positiv aus

(Mannheimer Morgen, 19. 5. 2004; „Klack“ - Koch/Schmidt lassen Junge/Wittmann keine Chance)

Halten wir nun fest: Nichtsdestotrotz kommt als Konnektor häufig vor, in konzessiver Funktion realisiert er sich eher im Mittelfeld, in der Nullposition dient er als Signal der Einleitung einer Interaktion, auch in geschriebenen Texten, die nicht umgangssprachlich markiert sind. Nichtsdestotrotz kann, trotz seiner Entstehungsgeschichte, in aktuellen Texten mit dem Tschechischen nicméně vor allem funktional gut korrespondieren. Wenn wir hier wieder zu der tschechischen Paralleldatenbank Treq zurückkehren, um diese Schlussfolgerung in der Übersetzungsrichtung Deutsch-Tschechisch zu überprüfen, ist der Treffer nicméně dann auch die erste Wahl. 


\section{Statt einer Zusammenfassung ein Plädoyer}

Aus diesen Beispielrecherchen lässt sich schlussfolgern, dass wir mit Methoden der sprachwissenschaftlichen Analyse nicht nur eine konkrete Äquivalenzlösung vorschlagen und argumentativ unterstützen können, viel wichtiger war jedoch zu zeigen, dass es konkrete korpusbasierte Instrumente waren, die uns zu diesem Ergebnis gebracht haben. Diese Überlegungen sollten somit als ein Plädoyer für die Berücksichtigung der korpuslinguistischen Kompetenzen in den Studieninhalten der übersetzungsorientierten Germanistik verstanden werden; dies wollen die folgenden Punkte verdeutlichen:

1. Die übersetzungsorientierte Germanistik als Teildisziplin der angewandten Sprachwissenschaft ist ein wichtiger Bestandteil der germanistischen Studienausrichtungen. Neben grundlegenden translatologischen Kompetenzen, die eine natürliche Priorität darstellen, geht es beim Aufbau einer sinnvollen Übersetzerausbildung um Vermittlung und Entwicklung von praktischen Kompetenzen, die dem angewandten Charakter eines translatologisch ausgerichteten Studiums entsprechen.

2. Die Korpuslinguistik im Allgemeinen vermittelt eine objektive Untersuchungsmethode; eine sinnvolle Arbeit mit korpuslinguistischen Instrumenten trägt zu unserer Sprachreflexion bei. Theoretische korpuslinguistische Untersuchungen, sowie konkrete praktische Recherchen, können bei werdenden Translatologen eine Grundlage bilden, auf die sie bei der Lösung konkreter vor allem die sprachliche Typik betreffender Übersetzungsprobleme zurückgreifen können.

3. Zur Aufzählung translatologisch relevanter Fragen gehört aber auch die einzelsprachliche Textkompetenz, d.h. das Wissen über die Typik der Gedankenführung mit entsprechenden Sprachgebrauchsmustern. Dieses Wissen wird durch eine konsequente Arbeit mit korpusbasierten Instrumenten aktiviert und forciert. Die Korpuslinguistik ist in diesem Sinne keine Theorie, sondern eine Methode, die in der Übersetzungspraxis helfen kann, wenn man bereits im Studium gelernt hat, wie sie tatsächlich helfen kann.

\section{Literatur}

BELICA, Cyril (2001): Kookkurrenzdatenbank CCDB. Eine korpuslinguistische Denk- und Experimentierplattform für die Erforschung und theoretische Begründung von systemisch-strukturellen Eigenschaften von Kohäsionsrelationen zwischen den Konstituenten des Sprachgebrauchs. 2001 ff., Institut für Deutsche Sprache, Mannheim.

BLUMENTHAL, Peter (1997): Sprachvergleich Deutsch-Französisch. 2. Neu erarbeitete und ergänzte Auflage. Tübingen: Niemeyer.

BREINDL, Eva (2011): Nach Rom freilich führen viele Wege. Zur Interaktion von Informationsstruktur, Diskursstruktur und Prosodie bei der Besetzung der Nacherstposition. In: Ferraresi, Gisella (Hrsg.). Konnektoren im Deutschen und im Sprachvergleich. Tübingen: Narr. S. 17-56. BUBENHOFER, Noah (2009): Sprachgebrauchsmuster. Korpuslinguistik als Methode der Diskursund Kulturanalyse. Berlin/New York: Walter de Gruyter. 
KLOSA, Annette (2011): elexiko - ein Bedeutungswörterbuch zwischen Tradition und Fortschritt. In: Sprachwissenschaft Jg. 36, H. 2/3, Heidelberg: Winter. S. 275-306.

KLOUDOVÁ, Věra/ŠEMELÍK, Martin (2013): Kookurenční databanka CCDB jako nástroj kontrastivní analýzy a překladu z češtiny do němčiny. In: Časopis pro moderní filologii 95, 2013, č. 1 , s. 39-51.

KRATOCHVÍlOVÁ (ZÜNDORF), Iva/WOLF, Norbert Richard (Hrsg.) (2010): Kompendium Korpuslinguistik. Heidelberg: Winter.

KRATOCHVÍLOVÁ (ZÜNDORF), Iva (2013): DeuCze.Von der Struktur und der Benutzbarkeit eines kleinen zweisprachigen Korpus. In: Kratochvílová (Zündorf), Iva/Wolf, Norbert Richard (Hrsg.) Grundlagen einer sprachwissenschaftlichen Quellenkunde. Tübingen: Narr. S. 15-27.

KRATOCHVÍLOVÁ (ZÜNDORF), Iva (2015): Kollokationen aus deutsch-tschechischer Perspektive: Kriterien und Optionen für die Erfassung der konfigurierten Mehrwortlexik. In: Ďurčo, Peter. Kollokationsforschung und Kollokationsdidaktik. Wien: Lit Verlag, 2016. S. 65-80.

MACHÁČKOVÁ, Eva (1990): Renesance výrazu nicméně, NŘ 73, s. 219-220.

PERKUHN, Rainer/KEIBEL, Holger/KUPIEC, Marc (2012): Korpuslinguistik. Paderborn: Fink. ROTHKEGEL, Annely (1994): Kollokationssbildung und Textbildung. In: Sandig, Barbara (1994) (ed.): Europhras 92. Tendenzen der Phraseologieforschung. Bochum, S. 499-523.

SCHANEN, François (2001): Textkonnektoren: Der begriffliche Hintergrund.“ In: Cambourian, Alain Textkonnektoren und andere textstrukturierende Einheiten.Tübingen: Stauffenburg (Eurogermanistik; 16), S. 1-18.

SLEZÁKOVÁ, Markéta (2002): Více, či méně? Nicméně! (aneb malá slova - velké změny) Naše řeč, ročník 85 (2002), číslo 4, s. 199-207.

ŠKRABAL, M. (2016): Paralelně - víceslovně - lépe? K možnostem nové verze databázepřekladových ekvivalentů Treq. In: Kolt 2016. Korpusy v kontrastivní lingvistice a translatologii. Praha: Ústav Českého národního korpusu. <http://ucnk.ff.cuni.cz/kolt2016/KOLT2016_abstrakty. pdf> [cit. 3. 4. 2017]

ŠTíCHA, František (2003): Česko-německá srovnávací gramatika. Praha: Argo.

ŠTíCHA, František (2013): Akademická gramatika spisovné češtiny. Praha: Academia.

TEUBERT, Wolfgang (1998): Korpus und Neologie. In Teubert, Wolfgang (Hg.) Neologie und Korpus. Tübingen: Narr. S. 129-170.

TEUBERT Wolfgang (1999): Korpuslinguistik und Lexikographie. In: Deutsche Sprache 4/1999, S. 292-313.

WASSNER, Ulrich Hermann (2011): Ausdrucksalternativen bei Konnektoren - Varianten oder Fehler? In: FERRARESI, Gisela (Hrsg.) Konnektoren im Deutsche und im Sprachvergleich. Beschreibung und grammatische Analyse. Tübingen: Narr, S. 57-90.

WOLF, Norbert Richard (1981). Am Beispiel Elias Canettis. Überlegungen zur Textsyntax und Texttypologie. In: Studien zur Literatur des 19. und 20. Jahrhunderts in Österreich. Fs. Alfred Doppler. Hg. von Johann HOLZNER/MICHAEL Klein/WOLFGANG Wiesmüller. Innsbruck 1981 (= Innsbrucker Beiträge zur Kulturwissenschaft, Germanistische Reihe 12), S. 205-218.

WOLF, Norbert Richard (2008): Textsyntax und/oder Textstilistik. In: FRITZ, Thomas A./ KOCH, Günter/TROST, Igor (Hrsg.): Literaturstil - sprachwissenschaftlich. Heidelberg: Winter, S. 57-69. 


\section{Elektronische Quellen}

ČNK https://kontext.korpus.cz/first_form https://wiki.korpus.cz/

CCDB http://corpora.ids-mannheim.de/ccdb/

Duden https://www.duden.de/woerterbuch

e-lexiko http://www.owid.de/wb/elexiko/start.html grammis https://grammis.ids-mannheim.de/konnektoren

Treq 2.0 http://treq.korpus.cz/

Prof. PhDr. Iva Zündorf, Ph.D. / izundorf@phil.muni.cz

Masarykova univerzita, Filozofická fakulta, Ústav germanistiky, nordistiky a nederlandistiky Arna Nováka 1, 60200 Brno, CZ 
